\title{
MULTI-COMPONENT NONLINEAR SCHRÖDINGER EQUATION ON SYMMETRIC SPACES WITH CONSTANT BOUNDARY CONDITIONS. PART I
}

\author{
VLADIMIR S. GERDJIKOV AND NIKOLAY A. KOSTOV
}

Communicated by Metin Gürses

Abstract. The multi-component nonlinear Schrödinger equations related to C.I $\simeq \mathrm{Sp}(2 p) / \mathrm{U}(p)$ and D.III $\simeq \mathrm{SO}(2 p) / \mathrm{U}(p)$-type symmetric spaces with non-vanishing boundary conditions are solvable by the inverse scattering method (ISM). We focus our attention on the single threshold case. We formulate the spectral properties of the Lax operator $L$, which is the generalized Zakharov-Shabat operator. Next we construct the corresponding fundamental analytic solutions (FAS) and adapt the Wronskian relations for the constant boundary conditions. They allow one to analyze the mapping from the class of allowed potentials $\mathcal{M}$ to each of the minimal sets of scattering data $\mathcal{T}_{i}, i=1,2$. The ISM for the Lax operator $L$ is interpreted as a nonlinear analog of the Fourier-transform method. As appropriate generalizations of the usual exponential functions we use the so-called 'squared solutions', which are constructed in terms of the FAS $\chi^{ \pm}(x, \lambda)$ of $L$ and the Cartan-Weyl basis of the Lie algebra, relevant to the symmetric space. We derive the completeness relation for the "squared solution" which turns out to provide the map from $\mathcal{M}$ to each $\mathcal{T}_{i}, i=1,2$. Such decompositions allow one to derive all fundamental properties of the multi-component nonlinear Schrödinger equations.

\section{Contents}

1 Introduction $\quad 2$

2 Spectral Properties of the Lax Operator $L$. The Single Threshold Case 5

2.1 The $t$-dependence of the Scattering Data . . . . . . . . . . 8

2.2 The FAS and the RHP . . . . . . . . . . . . . . . . 9

2.3 Constructing Singular Solutions of the RHP . . . . . . . . . . . . . . . . 14

2.4 The Resolvent of the Lax Operator . . . . . . . . . . . . . . . . 15

3 Wronskian Relations $\quad 18$ 
5 Discussions and Conclusions

\section{Introduction}

The integrability of the scalar nonlinear Schrödinger equation (NLS) with vanishing boundary conditions (v.b.c.)

$$
\mathrm{i} q_{t}+q_{x x}+2|q(x, t)|^{2} q(x, t)=0
$$

was discovered by Zakharov and Shabat in their pioneer work [29]. Soon after [30] Zakharov and Shabat proved the integrability and the physical importance of the NLS with constant boundary conditions (c.b.c.)

$$
\mathrm{i} q_{t}+2 q_{x x}-2\left(|q(x, t)|^{2}-\rho^{2}\right) q(x, t)=0, \quad \lim _{x \rightarrow \pm \infty} q(x, t)=q_{ \pm}
$$

where the asymptotic values $q_{ \pm}$satisfy $\left|q_{ \pm}\right|^{2}=\rho^{2}$. Notice the sign difference in the cubic nonlinearity as well as the additional term with the chemical potential $\rho$. Both versions of NLS equation served as models on which generalizations were made. The simplest non-trivial multi-component generalization of NLS is the vector NLS known as the Manakov model [22]

$$
\mathrm{i} \vec{q}_{t}+\bar{q}_{x x}+2\left(\vec{q}^{\dagger} \vec{q}(x, t)\right) \vec{q}(x, t)=0
$$

where $\vec{q}(x, t)$ is an $n$-component complex-valued vector vanishing fast enough for $x \rightarrow \pm \infty$. The c.b.c. version of vector NLS

$$
\mathrm{i} \vec{q}_{t}+\vec{q}_{x x}-\left(2\left(\vec{q}^{\dagger} \vec{q}(x, t)\right)-\rho^{2}\right) \vec{q}(x, t)+\left(\vec{q}_{ \pm}^{\dagger} \vec{q}(x, t)\right) \vec{q}_{ \pm}=0
$$

where $\lim _{x \rightarrow \pm \infty} \vec{q}(x, t)=\vec{q}_{ \pm}$and $\vec{q}_{-}=U_{0} \vec{q}_{+}$where $U_{0}$ is a constant unitary matrix also finds applications. Here $\rho^{2}=\vec{q}_{ \pm}^{\dagger} \vec{q}_{ \pm}$.

Equations (1) and (3) are particular cases of matrix NLS which is obtained from the system

$$
\begin{aligned}
\operatorname{iq} \mathbf{q}_{t}+\mathbf{q}_{x x}+2 \mathbf{q r q}(x, t) & =0 \\
-\mathrm{i} \mathbf{r}_{t}+\mathbf{r}_{x x}+2 \mathbf{r q r}(x, t) & =0
\end{aligned}
$$

after imposing an appropriate involution (reduction) compatible with the evolution of (5). Here $\mathbf{q}$ and $\mathbf{r}^{T}$ can be rectangular $n \times p$ matrix-valued functions of $x$ and $t$. One such involution is

$$
\mathbf{r}=B_{-} \mathbf{q}^{\dagger} B_{+}^{-1}, \quad B_{+}=\operatorname{diag}\left(\epsilon_{1}^{+}, \ldots, \epsilon_{p}^{+}\right), \quad B_{-}=\operatorname{diag}\left(\epsilon_{1}^{-}, \ldots, \epsilon_{n}^{-}\right)
$$


where $\left(\epsilon_{j}^{ \pm}\right)^{2}=1$, and the corresponding MNLS acquires the form

$$
\mathbf{i} \mathbf{q}_{t}+\mathbf{q}_{x x}+2 \mathbf{q} B_{-} \mathbf{q}^{\dagger} B_{+}^{-1} \mathbf{q}=0 .
$$

For $n=m=1$ and $r=q^{*}$ the system goes into the scalar NLS (1). For $m=1$ and $n>1$ and with appropriate choice of involution (6) the system is transformed into the Manakov model (3). All these versions are solvable with the ISM. The ISM is applicable to nonlinear evolution equations (NLEE) if they can be represented as compatibility condition of two linear problems [1,3, 16, 25, 28]

$$
[L(\lambda), M(\lambda)]=0
$$

which holds identically with respect to the spectral parameter $\lambda$.

The two linear operators $L(\lambda)$ and $M(\lambda)$ in the Zakharov-Shabat system (Z-Sh) for the MNLS on symmetric spaces associated with the simple Lie algebra $\mathfrak{g} \simeq C_{r}$ and $\mathfrak{g} \simeq D_{r}$ with v.b.c. are

$$
\begin{aligned}
L \psi & =\left(\mathrm{i} \frac{\partial}{\partial x}+Q(x, t)-\lambda J\right) \psi(x, t, \lambda)=0 \\
M \psi & =\left(\mathrm{i} \frac{\partial}{\partial t}+V_{2}(x, t)+\lambda V_{1}(x, t)-2 \lambda^{2} J\right) \psi(x, t, \lambda) \\
& =\psi(x, t, \lambda) C(\lambda) \\
Q(x, t) & =\left(\begin{array}{cc}
0 & \mathbf{q}(x, t) \\
\mathbf{r}(x, t) & 0
\end{array}\right), \quad J=\left(\begin{array}{cc}
1 & 0 \\
0 & -11
\end{array}\right)
\end{aligned}
$$

where $C(\lambda)$ is for now an arbitrary matrix-valued function which may depend only on $\lambda$ and will be fixed up below. The potential $Q(x, t)$ and $J$ are $2 r \times 2 r$ matrices with compatible block structure. Here

$$
V_{1}(x, t)=2 Q(x, t), \quad V_{2}(x, t)=\left[\operatorname{ad}_{J}^{-1} Q, Q\right]+2 \operatorname{iad}_{J}^{-1} Q_{x}(x, t)
$$

and $\operatorname{ad}_{J}^{-1}$ is the inverse of the adjoint action ad $J$ with respect to the element $J$, ad ${ }_{J} Y=[J, Y]$.

An effective tool to obtain new versions of multi-component nonlinear Schrödinger equations (MNLS) is the reduction group introduced by Mikhailov [23]. It allows one to impose algebraic constraints on the potential $Q(x, t)$ which are automatically compatible with the evolution. For example, the involution (6), which leads to MNLS with v.b.c. (7) is known as $\mathbb{Z}_{2}$-reduction and can be written as [13]

$$
B U^{\dagger}\left(x, t, \lambda^{*}\right) B^{-1}=U(x, t, \lambda)
$$


where $B$ is an automorphism of $\mathfrak{g}$ matrix such that $B^{2}=\mathbb{1},[J, B]=0$, and

$$
U(x, t, \lambda)=Q(x, t)-\lambda J .
$$

Below we analyze the MNLS

$$
\mathrm{iq} \mathbf{q}_{t}+\mathbf{q}_{x x}-2 \mathbf{q q} \mathbf{q}^{\dagger} \mathbf{q}+\mathbf{q} \bar{\mu}+\mu \mathbf{q}=0
$$

with constant boundary conditions (c.b.c.) at $x \rightarrow \pm \infty$

$$
\lim _{x \rightarrow \pm \infty} \mathbf{q}(x, t)=\mathbf{q}_{ \pm}, \quad \mu=\mathbf{q}_{+} \mathbf{q}_{+}^{\dagger}=\mathbf{q}_{-} \mathbf{q}_{-}^{\dagger}, \quad \bar{\mu}=\mathbf{q}_{+}^{\dagger} \mathbf{q}_{+}=\mathbf{q}_{-}^{\dagger} \mathbf{q}_{-}
$$

where $\mathbf{q}(x, t)$ in general can be a rectangular $n \times p$ matrix-valued function if we consider the symmetric spaces of class A.III [15]. Below we concentrate on the MNLS (15) related to the C.I and D.III-type symmetric spaces and therefore $\mathbf{q}(x, t)$ will be a square $r \times r$ matrix-valued function.

Its Lax pair is obtained from (9)-(12) by replacing $V_{2}(x, t)$ with

$$
V_{2}(x, t)=\left[\operatorname{ad}_{J}^{-1} Q, Q\right]+2 \operatorname{iad}_{J}^{-1} Q_{x}(x, t)-\left[\operatorname{ad}_{J}^{-1} Q_{ \pm}, Q_{ \pm}\right] .
$$

Here we have also imposed the additional condition $Q_{+}^{2}=Q_{-}^{2}$. It ensures that the two asymptotic Lax operators $L_{ \pm}=\mathrm{i} \partial_{x}+Q_{ \pm}-\lambda J$ have the same spectrum. It also ensures that the potential $V_{2}(x, t)$ in the second operator $M(\lambda)$ vanish for $x \rightarrow \pm \infty$. As a result the solutions of the MNLS equation (15) $\mathbf{q}(x, t)$ do not undergo strong oscillations with respect to time, see $[11,15]$.

Lax operators of the form (9) can be associated with each of the symmetric spaces listed below (for the definition see [18] and the Appendix). They are defined by specifying the simple Lie algebra $\mathfrak{g}$, having typical representation in $2 r \times 2 r$ matrices and the Cartan subalgebra element $J$

- C.I: $\mathfrak{g} \simeq C_{r} \simeq \mathfrak{s p}(2 r), J=H_{\vec{a}}$, where the vector $\vec{a}$ in the root space $\mathbb{E}^{r}$ dual to $J$ is given by $\vec{a}=\sum_{k=1}^{r} e_{k}$

- D.III : $\mathfrak{g} \simeq D_{r} \simeq \mathfrak{s o}(2 r), J=H_{\vec{a}}$, where the vector $\vec{a}$ in the root space $\mathbb{E}^{r}$ dual to $J$ is given by $\vec{a}=\sum_{k=1}^{r} e_{k}$.

Here the orthonormal vectors $e_{k}$ span the root space $\mathbb{E}^{r}$ of both types of algebras. The element $J$ belongs to the Cartan subalgebra $\mathfrak{h}$ and is dual to $\vec{a}$. Using $J$ we can split the set of positive roots into two two subsets $\triangle^{+}=\triangle_{0}^{+} \cup \triangle_{1}^{+}$. These sets, for the algebras that we are working with, are composed of the following roots

$$
\triangle_{0}^{+} \equiv\left\{e_{i}-e_{j}\right\}, \quad \triangle_{1}^{+} \equiv\left\{2 e_{i}, e_{i}+e_{j}\right\}, \quad 1 \leq i<j \leq r
$$


for $\mathfrak{g} \simeq \mathfrak{s p}(2 r)$ and

$$
\triangle_{0}^{+} \equiv\left\{e_{i}-e_{j}\right\}, \quad \triangle_{1}^{+} \equiv\left\{e_{i}+e_{j}\right\}, \quad 1 \leq i<j \leq r
$$

for $\mathfrak{g} \simeq \mathfrak{s o}(2 r)$.

The root vectors of the algebra are denoted by $E_{\alpha}$ where $\alpha$ is the corresponding root. We will need also the projector $P_{J}=\operatorname{ad}_{J}^{-1}$ ad ${ }_{J}$ onto the co-adjoint orbit $O_{J}$ of the element $J$. Here the inverse of the adjoint action is $\operatorname{ad}_{J}^{-1} Y=\frac{1}{2} J Y$. The generic element of $X \in O_{J}$ is the one that satisfies the relation $X=P_{J} X$. Obviously the potential of the Z-Sh system $Q(x, t)$ and its variation $\delta Q(x, t)$ belong to $O_{J}$.

This paper extends the results of $[11,12,15]$ and [4]. In Section 2 we analyze the spectral properties of the generalized Z-Sh operator with c.b.c. We start with the single threshold case for which $Q_{+}^{2}=Q_{-}^{2}=\rho^{2} \mathbb{1}$. First we construct the fundamental analytic solutions (FAS) and formulate the corresponding RiemannHilbert problem (RHP) on a Riemannian surface. We also derive the time evolution of the scattering matrix. Next we briefly analyze the construction of singular solutions for the RHP and then propose an explicit formula for the kernel of the resolvent $R^{ \pm}(x, y, \lambda)$ of $L$ in terms of the FAS. Then we apply the contour integration method and derive the spectral decomposition for $L$. In Section 3 we derive the Wronskian relations which are the basic tool for analyzing the mapping $\mathcal{F}: \mathcal{M} \rightarrow \mathcal{T}_{i}$. We also introduce minimal sets of scattering data $\mathcal{T}_{i}, i=1,2$. In Section 4 we derive the completeness relation for the "squared solutions" of the Lax operator generalizing the results of $[20,21]$. Thus we prove that the ISM is equivalent to a generalized Fourier transform also for the Lax operators with c.b.c. Thus we have shown that the nonlinear evolution of equation (15) transforms into linear one in terms of the scattering data of $L$. In the last Section 5 we briefly consider the generic multi-threshold case when $Q_{+}^{2}=Q_{-}^{2}$ have $2 r$ different eigenvalues $\pm \rho_{j}^{2}$ and draw some conclusions.

\section{Spectral Properties of the Lax Operator $L$. The Single Threshold Case}

The spectrum of the asymptotic operators $L_{ \pm}$is purely continuous and is determined by the eigenvalues of $Q_{ \pm}$which generically may be arbitrary complex numbers. However, here we consider only the case when $L$ becomes self-adjoint. As a result its potential $Q(x, t)$ acquires the form

$$
Q(x, t)=-Q^{\dagger}(x, t), \quad Q(x, t)=\left(\begin{array}{cc}
0 & \mathbf{q}(x, t) \\
-\mathbf{q}^{\dagger}(x, t) & 0
\end{array}\right) .
$$


For simplicity reasons we will consider only the case when all of the eigenvalues of the asymptotic matrices $Q_{ \pm}$are real and equal

$$
\rho_{1}=\rho_{2}=\cdots=\rho_{r}=\rho>0
$$

where $\rho$ is real parameter. It determines the threshold, or equivalently, the chemical potential of the MNLS. As a result we have the following condition on the eigenvalues of the asymptotic matrices [15] $\mathbf{q}_{ \pm} \mathbf{q}_{ \pm}^{\dagger}(x, t)=\rho^{2} \mathbb{1}$ and the correspondence with the isotropic problem is obvious: $\mu=\bar{\mu}=\rho^{2} 11$.

The requirement that the potentials of the Z-Sh system belong to $\mathfrak{g}$ can be formulated as a reduction condition [7,23]

$$
\begin{aligned}
& S_{0}^{-1} U^{T}(x, t, \lambda) S_{0}=-U(x, t, \lambda), \\
& S_{0}^{-1} V^{T}(x, t, \lambda) S_{0}=-V(x, t, \lambda), \quad S_{0}^{-1} J S_{0}=-J
\end{aligned}
$$

which has trivial action on $\lambda$. The matrix $S_{0}$ is the one which realizes the definition of the algebras $C_{r} \simeq \mathfrak{s p}(2 r)$ or $D_{r} \simeq \mathfrak{s o}(2 r)$ in the typical representation [7,18] . In what follows we will define the Lie algebra $\mathfrak{g}$ by

$$
\mathfrak{g} \equiv\left\{X ; X+S_{0}^{-1} X^{t} S_{0}=0\right\}
$$

where

$$
S_{0}=\sum_{s=1}^{r}(-1)^{s+1}\left(E_{s \bar{s}}-E_{\bar{s} s}\right)
$$

for $\mathfrak{g} \simeq \mathfrak{s p}(2 r)$ and

$$
S_{0}=\sum_{s=1}^{r}(-1)^{s+1}\left(E_{s \bar{s}}+E_{\bar{s} s}\right)
$$

for $\mathfrak{g} \simeq \mathfrak{s o}(2 r)$. Here $\bar{s}=2 r-s+1$ and $E_{k s}$ are $2 r \times 2 r$ matrices, defined by $\left(E_{k s}\right)_{i j}=\delta_{k i} \delta_{s j}$. Note that $S_{0}^{2}=\epsilon_{0} \mathbb{1}$, where $\epsilon_{0}=-1$ for $\mathfrak{s p}(2 r)$ and $\epsilon_{0}=1$ for $\mathfrak{s o}(2 r)$.

The reduction (22) imposes restrictions only on the coefficients of $Q(x, t)$ such that for $C_{r} \simeq \mathfrak{s p}(2 r)$ we can put

$$
Q(x, t)=\sum_{i<j}\left(q_{i j} E_{e_{i}+e_{j}}-q_{j i}^{*} E_{-e_{i}-e_{j}}\right)+\sum_{i=1}^{r}\left(q_{i} E_{2 e_{i}}-q_{i}^{*} E_{-2 e_{i}}\right)
$$

while in the $D_{r} \simeq \mathfrak{s o}(2 r)$-case we have

$$
Q(x, t)=\sum_{i<j}\left(q_{i j} E_{e_{i}+e_{j}}-q_{j i}^{*} E_{-e_{i}-e_{j}}\right)
$$


where the ${ }^{*}$ means a complex conjugation. The definitions of the root vectors $E_{\alpha}$ can be found in the Appendix. In the typical representations of $C_{r}$ and $D_{r}$ these choices for $Q(x, t)$ have always the block structure shown in (20). For example, in the case of $\mathfrak{g} \simeq \mathfrak{s p}(4)$ the block $\mathbf{q}$ is parametrized by three functions

$$
\mathbf{q}(x, t)=\left(\begin{array}{cc}
q_{12} & \sqrt{2} q_{1} \\
\sqrt{2} q_{2} & -q_{12}
\end{array}\right) .
$$

The corresponding sets of MNLS for these choices of $Q(x, t)$ and v.b.c. were first derived in [7]. For c.b.c. with $\mathbf{r}=-\mathbf{q}^{\dagger}$ MNLS take the form (15) with the additional linear in $\mathbf{q}$ terms ensuring regular behavior of the solutions for $t \rightarrow$ $\pm \infty$.

Let us outline the construction of the FAS. In the particular case that we are considering - the isotropic problem - the Jost solutions are defined as fundamental solutions with fixed asymptotic for $x \rightarrow \pm \infty$

$$
\lim _{x \rightarrow \infty} \psi(x, \lambda) \mathrm{e}^{\mathrm{i} \mu(\lambda) J x}=\psi_{0}(\lambda), \quad \lim _{x \rightarrow-\infty} \phi(x, \lambda) \mathrm{e}^{\mathrm{i} \mu(\lambda) J x}=\phi_{0}(\lambda)
$$

where $2 r \times 2 r$ matrices $\psi_{0}(\lambda)$ and $\phi_{0}(\lambda)$ take value in the corresponding group $\mathcal{G}$ and diagonalize the potential of the Lax operator $L$

$$
\begin{aligned}
& \left(Q_{+}-\lambda J\right) \psi_{0}(\lambda)=-\psi_{0}(\lambda) \mu(\lambda) J \\
& \left(Q_{-}-\lambda J\right) \phi_{0}(\lambda)=-\phi_{0}(\lambda) \mu(\lambda) J
\end{aligned}
$$

where $\mu(\lambda)=\sqrt{\lambda^{2}-m^{2}}$. They have the block structure

$$
\psi_{0}(\lambda)=\left(\begin{array}{cc}
\underline{A} & S_{1} \underline{B} \\
\underline{B} S_{1} & \underline{A}
\end{array}\right), \quad \phi_{0}(\lambda)=V_{0}\left(\begin{array}{cc}
\underline{A} & S_{1} \underline{B} \\
\underline{B} S_{1} & \underline{A}
\end{array}\right) .
$$

The $r \times r$ matrices $\underline{\mathrm{A}}, \underline{\mathrm{B}}$ and $S_{1}$ are given by

$$
\underline{A}=\sqrt{\frac{\lambda+\mu(\lambda)}{2 \mu(\lambda)}}, \quad \underline{B}=\sqrt{\frac{\lambda-\mu(\lambda)}{2 \mu(\lambda)}}, \quad S_{1}=\sum_{s=1}^{r} e_{s, r-s+1}
$$

where $e_{p q}$ are $r \times r$ matrices such that $\left(e_{p q}\right)_{i j}=\delta_{i p} \delta_{j q}$ and the phase factor $V_{0}$ is $2 r \times 2 r$ diagonal and unitary matrix of the form $V_{0}=\exp \left(\mathrm{i} \varphi_{0} J\right)$.

Given the potential $Q(x)$ one can obtain the Jost solutions uniquely. The Jost solutions in turn determine uniquely the scattering matrix $T(\lambda)$ and its inverse $\widehat{T}(\lambda)$. $Q(x)$ contains at most $\left|\triangle_{+}^{1}\right|$ independent complex-valued functions of $x$. Thus it is natural to expect that at most $\left|\triangle_{1}^{+}\right|$of the coefficients of $T(\lambda)$ for $\lambda \in R_{m}$, 
instead of $(2 r)^{2}$, will be independent. Here $\left|\triangle_{1}^{+}\right|$is the number of roots in $\triangle_{1}^{+}$, i.e. $\left|\triangle_{1}^{+}\right|=r(r+1) / 2$ for $C_{r}$ and $\left|\triangle_{1}^{+}\right|=r(r-1) / 2$ for $D_{r}$. The continuous spectrum $R_{m}=(-\infty,-m) \cup(m, \infty)$ is determined by the condition $|\lambda| \geq m$.

The two Jost solutions are fundamental solutions and must be linearly dependent. This means that there exists a matrix $T(t, \lambda)$, called scattering matrix, which connects them and has an appropriate block structure.

$$
T(t, \lambda)=\psi^{-1}(x, t, \lambda) \phi(x, t, \lambda) .
$$

\subsection{The $t$-dependence of the Scattering Data}

A direct consequence of the Lax representation is the evolution law for the scattering matrix. Indeed, let us consider the regularized $M$-operator (i.e. $M$ defined by equation (10) but with $V_{2}$ given by equation (17)) acting on the Jost solution $\phi(x, t, \lambda)$. First we will define the function $C(\lambda)$ by requesting that the asymptotic of $\phi(x, t, \lambda)$ for $x \rightarrow-\infty$ (see equation (27) holds true for any $t$. Taking in it the $x \rightarrow-\infty$ limit and taking into account that $\lim _{x \rightarrow \pm \infty} V_{2}(x, t)=0$ (see equation (17)) we get:

$$
2 \lambda\left(Q_{-}-\lambda J\right) \phi_{0}(\lambda) \mathrm{e}^{\mathrm{i} \mu(\lambda) J x}=\phi_{0}(\lambda) \mathrm{e}^{\mathrm{i} \mu(\lambda) J x} C(\lambda)
$$

which combined with equation (28) means that

$$
C(\lambda)=-2 \lambda \mu(\lambda) J .
$$

Taking the limit to $x \rightarrow \infty$ we obtain

$$
\begin{aligned}
\mathrm{i} \psi_{0}(\lambda) \mathrm{e}^{\mathrm{i} \mu(\lambda) J x} \frac{\mathrm{d} T}{\mathrm{~d} t}+2 \lambda\left(Q_{+}-\lambda J\right) \psi_{0}(\lambda) \mathrm{e}^{\mathrm{i} \mu(\lambda) J x} T(t, \lambda) & \\
& =\psi_{0}(\lambda) \mathrm{e}^{\mathrm{i} \mu(\lambda) J x} T(t, \lambda) C(\lambda)
\end{aligned}
$$

i.e. the $t$-dependence of the scattering matrix is given by

$$
\mathrm{i} \frac{\mathrm{d} T}{\mathrm{~d} t}-2 \lambda \mu(\lambda)[J, T(t, \lambda)]=0 .
$$

Thus we find that the MNLS with c.b.c. in the case of single threshold has the same dispersion law $f(\lambda)=2 \lambda \mu(\lambda)$ as the scalar NLS with c.b.c., see [25].

We can use for the scattering matrix the same block-matrix structure as in v.b.c. case [15]

$$
\begin{aligned}
\phi(x, \lambda) & =\psi(x, \lambda) T(\lambda), \\
T(\lambda) & =\left(\begin{array}{cc}
\mathbf{a}^{+}(\lambda) & -\mathbf{b}^{-}(\lambda) \\
\mathbf{b}^{+}(\lambda) & \mathbf{a}^{-}(\lambda)
\end{array}\right), \quad \widehat{T}(\lambda)=\left(\begin{array}{cc}
\mathbf{c}^{-}(\lambda) & \mathbf{d}^{-}(\lambda) \\
-\mathbf{d}^{+}(\lambda) & \mathbf{c}^{+}(\lambda)
\end{array}\right) .
\end{aligned}
$$


The block matrices of the inverse matrix are defined as follows

$$
\begin{aligned}
& \mathbf{c}^{-}(\lambda)=\widehat{\mathbf{a}}^{+}(\lambda)\left(\mathbb{1}+\rho^{-} \rho^{+}\right)^{-1}=\left(\mathbb{1}+\tau^{+} \tau^{-}\right)^{-1} \widehat{\mathbf{a}}^{+}(\lambda) \\
& \mathbf{d}^{-}(\lambda)=\widehat{\mathbf{a}}^{+}(\lambda) \rho^{-}(\lambda)\left(\mathbb{1}+\rho^{+} \rho^{-}\right)^{-1}=\left(\mathbb{1}+\tau^{+} \tau^{-}\right)^{-1} \tau^{+}(\lambda) \widehat{\mathbf{a}}^{-}(\lambda) \\
& \mathbf{c}^{+}(\lambda)=\widehat{\mathbf{a}}^{-}(\lambda)\left(\mathbb{1}+\rho^{+} \rho^{-}\right)^{-1}=\left(\mathbb{1}+\tau^{+} \tau^{-}\right)^{-1} \widehat{\mathbf{a}}^{-}(\lambda) \\
& \mathbf{d}^{+}(\lambda)=\widehat{\mathbf{a}}^{-}(\lambda) \rho^{+}(\lambda)\left(\mathbb{1}+\rho^{-} \rho^{+}\right)^{-1}=\left(\mathbb{1}+\tau^{-} \tau^{+}\right)^{-1} \tau^{-}(\lambda) \widehat{\mathbf{a}}^{+}(\lambda)
\end{aligned}
$$

where

$$
\begin{aligned}
\rho^{ \pm}(\lambda) & =\mathbf{b}^{ \pm}(\lambda) \widehat{\mathbf{a}}^{ \pm}(\lambda)=\widehat{\mathbf{c}}^{ \pm}(\lambda) \mathbf{d}^{ \pm}(\lambda) \\
\tau^{ \pm}(\lambda) & =\widehat{\mathbf{a}}^{ \pm}(\lambda) \mathbf{b}^{\mp}(\lambda)=\mathbf{d}^{\mp}(\lambda) \widehat{\mathbf{c}}^{ \pm}(\lambda)
\end{aligned}
$$

are the multi-component generalizations of the reflection $\rho^{ \pm}, \tau^{ \pm}$coefficients for the scalar case, see $[27,29,30]$.

From equation (35) there follows that the diagonal blocks of $T(t, \lambda)$ and its inverse are time independent

$$
\mathrm{i} \frac{\mathrm{d} \mathbf{a}^{ \pm}}{\mathrm{d} t}=0, \quad \mathrm{i} \frac{\mathrm{d} \mathbf{c}^{ \pm}}{\mathrm{d} t}=0 .
$$

In other words these blocks can be used as generating functionals of the conservation laws of the MNLS. The off-diagonal blocks of $T(t, \lambda)$ and its inverse evolve in time according to

$$
\mathrm{i} \frac{\mathrm{d} \mathbf{b}^{ \pm}}{\mathrm{d} t} \mp 2 \lambda \mu(\lambda) \mathbf{b}^{ \pm}=0, \quad \mathrm{i} \frac{\mathrm{d} \mathbf{d}^{ \pm}}{\mathrm{d} t} \mp 2 \lambda \mu(\lambda) \mathbf{d}^{ \pm}=0 .
$$

As a consequence for the reflection coefficients $\rho^{ \pm}$and $\tau^{ \pm}$we get

$$
\mathrm{i} \frac{\mathrm{d} \rho^{ \pm}}{\mathrm{d} t} \mp 2 \lambda \mu(\lambda) \rho^{ \pm}=0, \quad \mathrm{i} \frac{\mathrm{d} \tau^{ \pm}}{\mathrm{d} t} \pm 2 \lambda \mu(\lambda) \tau^{ \pm}=0 .
$$

\subsection{The FAS and the RHP}

First we will write down the integral equations which are satisfied by the Jost solutions. It will be more convenient to introduce

$$
\begin{aligned}
& X_{+}(x, \lambda)=\psi_{0}^{-1} \psi(x, \lambda) \mathrm{e}^{\mathrm{i} J_{0}(\lambda) x} \\
& X_{-}(x, \lambda)=\phi_{0}^{-1} \phi(x, \lambda) \mathrm{e}^{\mathrm{i} J_{0}(\lambda) x}
\end{aligned}
$$

which satisfy $\lim _{x \rightarrow \pm \infty} X_{ \pm}(x, \lambda)=1$. It is not difficult to show that they must satisfy the following integral equations

$$
X_{+}(x, \lambda)=\mathbb{1}+\mathrm{i} \int_{\infty}^{x} \mathrm{~d} y \mathrm{e}^{\mathrm{i} \mu(\lambda) J(y-x)} U_{+}(x, \lambda) X_{+}(y, \lambda) \mathrm{e}^{-\mathrm{i} \mu(\lambda) J(y-x)}
$$


and

$$
X_{-}(x, \lambda)=\mathbb{1}+\mathrm{i} \int_{-\infty}^{x} \mathrm{~d} y \mathrm{e}^{\mathrm{i} \mu(\lambda) J(y-x)} U_{-}(x, \lambda) X_{-}(y, \lambda) \mathrm{e}^{-\mathrm{i} \mu(\lambda) J(y-x)}
$$

where

$$
\begin{aligned}
& U_{+}(x, \lambda)=\psi_{0}^{-1}(\lambda)\left(Q(x)-Q_{+}\right) \psi_{0}(\lambda) \\
& U_{-}(x, \lambda)=\phi_{0}^{-1}(\lambda)\left(Q(x)-Q_{-}\right) \phi_{0}(\lambda) .
\end{aligned}
$$

Below we will use the following block structure for $X_{ \pm}(x, \lambda)$, each block being $r \times r$ matrix

$$
\left|X_{ \pm, 1}(x, \lambda)\right\rangle=\left(\begin{array}{c}
X_{ \pm, 11} \\
X_{ \pm, 21}
\end{array}\right), \quad\left|X_{ \pm, 2}(x, \lambda)\right\rangle=\left(\begin{array}{c}
X_{ \pm, 12} \\
X_{ \pm, 22}
\end{array}\right) .
$$

Then from equation (43) we obtain the following integral equations for the functions $\left|X_{+, 1}(x, \lambda)\right\rangle$ and $\left|X_{+, 2}(x, \lambda)\right\rangle$

$$
\begin{aligned}
\left|X_{+, 1}(x, \lambda)\right\rangle & =\left(\begin{array}{l}
1 \\
0
\end{array}\right)+\mathrm{i} \int_{\infty}^{x} \mathrm{~d} y\left(\begin{array}{c}
\left(U_{+}(x, \lambda) X_{+}(y, \lambda)\right)_{11} \\
\mathrm{e}^{-2 \mathrm{i} \mu(\lambda)(y-x)}\left(U_{+}(x, \lambda) X_{+}(y, \lambda)\right)_{21}
\end{array}\right) \\
\left|X_{+, 2}(x, \lambda)\right\rangle & =\left(\begin{array}{l}
0 \\
1
\end{array}\right)+\mathrm{i} \int_{\infty}^{x} \mathrm{~d} y\left(\begin{array}{c}
\left(\mathrm{e}^{2 \mathrm{i} \mu(\lambda)(y-x)} U_{+}(x, \lambda) X_{+}(y, \lambda)\right)_{12} \\
\left(U_{+}(x, \lambda) X_{+}(y, \lambda)\right)_{22}
\end{array}\right) .
\end{aligned}
$$

These are Volterra-type integral equations. It is well known that such equations allow solutions if one can prove that the integral is convergent. For $\operatorname{Im} \mu(\lambda)<0$ the convergence of the integral in equation (47) follows from the following facts: i) $Q(x)-Q_{+}$tends to zero fast enough for $x \rightarrow \infty$; ii) the exponential factor $\exp (-2 \mathrm{i} \mu(\lambda)(y-x))$ decreases exponentially. Thus we have outlined the proof of the fact that $\left|X_{+, 1}(x, \lambda)\right\rangle$ is an analytic function of $\lambda$ for $\operatorname{Im} \mu(\lambda)<0$. Similarly, one can prove that $\left|X_{+, 2}(x, \lambda)\right\rangle$ is an analytic function of $\lambda$ for $\operatorname{Im} \mu(\lambda)>0$.

The same ideas can be applied also to the integral equations for $X_{-}(x, \lambda)$.

From the above arguments it is also obvious that the analyticity properties hold true on one of the sheets of the two-sheeted Riemannian surface $\mathcal{S}$

$$
\mathcal{S}=\mathcal{S}_{1} \cup \mathcal{S}_{2}
$$

associated with the square root $\mu(\lambda)$. The relevant cut is

$$
C_{\rho} \equiv[-\infty \leq \operatorname{Re} \lambda \leq-\rho] \cup[\rho \leq \operatorname{Re} \lambda \leq \infty]
$$

Each sheet of this surface is determined by the sign of $\mu(\lambda)$, i.e.,

$$
\mathcal{S}_{1}: \operatorname{Im} \mu(\lambda)>0, \quad \mathcal{S}_{2}: \operatorname{Im} \mu(\lambda)<0 .
$$


From equations (29)-(30) it follows that $\psi_{0}(\lambda)$ and $\phi_{0}(\lambda)$ are also analytic on both sheets $S_{j}$ having singularities only on the end-points of the cuts.

As a consequence we can formulate the analyticity properties of the Jost solutions as follows

$$
\psi(x, \lambda)=\left(\left|\psi^{-}(x, \lambda)\right\rangle,\left|\psi^{+}(x, \lambda)\right\rangle\right), \quad \phi(x, \lambda)=\left(\left|\phi^{+}(x, \lambda)\right\rangle,\left|\phi^{-}(x, \lambda)\right\rangle\right)
$$

where $\left|\psi^{ \pm}\right\rangle$and $\left|\psi^{ \pm}\right\rangle$denote a $r \times 2 r$ matrix composed of the corresponding $r$ columns of the Jost solutions. The superscript + (respectively - ) means analyticity on the first sheet $\mathcal{S}_{1}$ (respectively on the second sheet $\mathcal{S}_{2}$ ). We will use similar representations for their inverse

$$
\widehat{\psi}(x, \lambda)=\left(\begin{array}{c}
\left\langle\widehat{\psi}^{+}(x, \lambda)\right| \\
\left\langle\widehat{\psi}^{-}(x, \lambda)\right|
\end{array}\right), \quad \widehat{\phi}(x, \lambda)=\left(\begin{array}{c}
\left\langle\widehat{\phi}^{-}(x, \lambda)\right| \\
\left\langle\widehat{\phi}^{+}(x, \lambda)\right|
\end{array}\right)
$$

where again the superscripts + and - refer to the analyticity properties.

Next, we can construct FAS on each of the sheets by simply combining the blocks of the Jost solutions with the same analyticity properties

$$
\chi^{+}(x, \lambda) \equiv\left(\left|\phi^{+}\right\rangle,\left|\psi^{+}\right\rangle\right)(x, \lambda), \quad \chi^{-}(x, \lambda) \equiv\left(\left|\psi^{-}\right\rangle,\left|\phi^{-}\right\rangle\right)(x, \lambda) .
$$

They are related to the Jost solutions by upper- (respectively lower-) block-triangular functions $\mathbf{S}^{ \pm}$and $\mathbf{T}^{ \pm}$(see [10] for similar decompositions in the v.b.c. case)

$$
\chi^{ \pm}(x, \lambda)=\psi(x, \lambda) \mathbf{T}^{\mp}=\phi(x, \lambda) \mathbf{S}^{ \pm}
$$

where

$$
\begin{aligned}
\mathbf{S}^{+} & =\left(\begin{array}{cc}
11 & \mathbf{d}^{-} \\
0 & \mathbf{c}^{+}
\end{array}\right), & \mathbf{T}^{-} & =\left(\begin{array}{cc}
\mathbf{a}^{+} & 0 \\
\mathbf{b}^{+} & \mathbb{1}
\end{array}\right) \\
\mathbf{S}^{-} & =\left(\begin{array}{cc}
\mathbf{c}^{-} & 0 \\
-\mathbf{d}^{+} & 1
\end{array}\right), & \mathbf{T}^{+} & =\left(\begin{array}{cc}
1 & -\mathbf{b}^{-} \\
0 & \mathbf{a}^{-}
\end{array}\right) .
\end{aligned}
$$

These triangular factors are directly related to the generalized Gauss decompositions of the $T(\lambda)$. Indeed they satisfy

$$
T(\lambda)=\mathbf{T}^{-}(\lambda) \widehat{\mathbf{S}}^{+}(\lambda)=\mathbf{T}^{+}(\lambda) \widehat{\mathbf{S}}^{-}(\lambda) .
$$

Here and after the hat ${ }^{-}$means taking the inverse matrix.

The proper generalized Gauss decompositions involve block-triangular factors that have unit elements on the diagonal. Simple rearrangements do the job as 
follows

$$
\begin{array}{lll}
\mathbf{S}^{+}=S^{ \pm} \mathcal{D}_{1}^{ \pm}, & S_{J}^{+}=\left(\begin{array}{cc}
\mathbb{1} & \tau^{+} \\
0 & \mathbb{1}
\end{array}\right), & S_{J}^{-}=\left(\begin{array}{cc}
\mathbb{1} & 0 \\
-\tau^{-} & \mathbb{1}
\end{array}\right) \\
\mathbf{T}^{+}=T^{ \pm} \mathcal{D}_{2}^{ \pm}, & T_{J}^{+}=\left(\begin{array}{cc}
\mathbb{1} & -\rho^{-} \\
0 & \mathbb{1}
\end{array}\right), & T_{J}^{-}=\left(\begin{array}{cc}
\mathbb{1} & 0 \\
\rho^{+} & \mathbb{1}
\end{array}\right) .
\end{array}
$$

The block-diagonal matrices $\mathcal{D}_{j}^{+}$(respectively $\mathcal{D}_{j}^{-}$), $j=1,2$, are given by

$$
\begin{array}{ll}
\mathcal{D}_{1}^{+}=\left(\begin{array}{cc}
\mathbb{1} & 0 \\
0 & \mathbf{c}^{+}
\end{array}\right), & \mathcal{D}_{2}^{+}=\left(\begin{array}{cc}
\mathbf{a}^{+} & 0 \\
0 & \mathbb{1}
\end{array}\right) \\
\mathcal{D}_{1}^{-}=\left(\begin{array}{cc}
\mathbf{c}^{-} & 0 \\
0 & \mathbb{1}
\end{array}\right), & \mathcal{D}_{2}^{-}=\left(\begin{array}{cc}
\mathbb{1} & 0 \\
0 & \mathbf{a}^{-}
\end{array}\right) .
\end{array}
$$

Therefore the proper generalized Gauss decomposition takes the form

$$
T(\lambda)=T_{J}^{-} \mathcal{D}_{J}^{+} \widehat{S}_{J}^{+}=T_{J}^{+} \mathcal{D}_{J}^{-} \widehat{S}_{J}^{-}
$$

where

$$
\mathcal{D}_{J}^{+}=\mathcal{D}_{1}^{+} \mathcal{D}_{2}^{+}=\left(\begin{array}{cc}
\mathbf{a}^{+} & 0 \\
0 & \mathbf{c}^{+}
\end{array}\right), \quad \mathcal{D}_{J}^{-}=\mathcal{D}_{1}^{-} \mathcal{D}_{2}^{-}=\left(\begin{array}{cc}
\mathbf{c}^{-} & 0 \\
0 & \mathbf{a}^{-}
\end{array}\right) .
$$

Using the properties of the symmetric spaces and the Cartan-Weyl basis one can write down the triangular factors also as

$$
\begin{aligned}
& S_{J}^{ \pm}(\lambda)=\exp \left( \pm \sum_{\alpha \in \Delta_{1}^{+}} \tau_{\alpha}^{ \pm}(\lambda) E_{ \pm \alpha}\right)=\mathbb{1}+\sum_{\alpha \in \Delta_{1}^{+}} \tau_{\alpha}^{ \pm}(\lambda) E_{ \pm \alpha} \\
& T_{J}^{\mp}(\lambda)=\exp \left( \pm \sum_{\alpha \in \Delta_{1}^{+}} \rho_{\alpha}^{ \pm}(\lambda) E_{ \pm \alpha}\right)=\mathbb{1}+\sum_{\alpha \in \Delta_{1}^{+}} \rho_{\alpha}^{ \pm}(\lambda) E_{ \pm \alpha} .
\end{aligned}
$$

Now we redefine the FAS $\chi^{ \pm}(x, \lambda)$ from equation (53) by

$$
\chi^{ \pm}(x, \lambda)=\phi(x, \lambda) S_{J}^{ \pm}=\psi(x, \lambda) T_{J}^{\mp} \mathcal{D}_{J}^{ \pm}(\lambda) .
$$

After this redefinition $\chi^{ \pm}(x, \lambda)$ take values in the corresponding Lie group.

We also introduce the FAS $\xi^{ \pm}(x, \lambda)$ of the related linear problem

$$
\mathrm{i} \frac{\mathrm{d} \xi^{ \pm}}{\mathrm{d} x}+(Q(x)-\lambda J) \xi^{ \pm}(x, \lambda)+\xi^{ \pm}(x, \lambda) \mu(\lambda) J=0
$$


which are expressed in terms of the Jost solutions by

$$
\xi^{ \pm}(x, \lambda)=\phi(x, \lambda) S_{J}^{ \pm}(\lambda) \mathrm{e}^{\mathrm{i} \mu(\lambda) J x}=\psi(x, \lambda) T_{J}^{\mp}(\lambda) \mathcal{D}_{J}^{ \pm}(\lambda) \mathrm{e}^{\mathrm{i} \mu(\lambda) J x}
$$

for $\lambda \in \mathfrak{R}$. These FAS satisfy the following RHP

$$
\xi^{+}(x, \lambda)=\xi^{-}(x, \lambda) G(x, \lambda), \quad G(x, \lambda)=\mathrm{e}^{-\mathrm{i} \mu(\lambda) J x} \widehat{S}^{-} S^{+}(\lambda) \mathrm{e}^{\mathrm{i} \mu(\lambda) J x}
$$

on the Riemannian surface $\mathcal{S}$. Of course reducing the ISP for the Lax operator $L$ to an RHP allows one to apply the dressing Zakharov-Shabat method. Of course, in order to render the problem uniquely solvable we need to specify also the normalization condition for $\xi^{ \pm}(x, \lambda)$ which takes the form

$$
\lim _{\lambda \rightarrow \infty, \lambda \in \mathcal{R}_{1}} \xi^{+}(x, \lambda)=\mathbb{1} .
$$

The possibility to reduce the inverse scattering problem to an RHP allows one to apply the dressing Zakharov-Shabat method [31,32] for evaluating the soliton solutions, for A.III-type symmetric spaces see [15].

We will say that $\xi^{ \pm}(x, \lambda)$ are regular solutions of the RHP (64) if $\xi^{+}(x, \lambda)$ has no singularities for any $\lambda \in \mathcal{R}_{+}$and $\xi^{-}(x, \lambda)$ has no singularities for any $\lambda \in \mathcal{R}_{-}$. The character of these singularities in terms of $\lambda$ may be rather involved. The problem simplifies if we introduce the uniformizing variable $z$ on the surface $\mathcal{R}$

$$
z=\frac{\lambda+\sqrt{\lambda^{2}-\rho^{2}}}{2 \rho}, \quad \frac{1}{z}=\frac{\lambda-\sqrt{\lambda^{2}-\rho^{2}}}{2 \rho} .
$$

The change of variables from $\lambda$ to $z$ is a conformal transformation which maps the sheets $\mathcal{R}_{1}$ and $\mathcal{R}_{1}$ into $z \in \mathbb{C}_{+}$and $z \in \mathbb{C}_{-}$. The advantage of this change of variables is that it becomes easier to treat the singularities of $\xi^{ \pm}(x, \lambda)$. A more thorough analysis of the structure of the zeroes of $\mathbf{a}^{ \pm}$and $\mathbf{c}^{ \pm}$shows that, like in the scalar NLS case [25], the FAS $\xi^{ \pm}(x, \lambda)$ as functions of $z$ allow pole singularities in $z$. In what follows we assume that these poles are simple, which means that

$$
\xi^{ \pm}(x, z)=\frac{\xi_{k}^{ \pm}(x)}{z-z_{k}^{ \pm}}+\dot{\xi}_{k}^{ \pm}(x)+\mathcal{O}\left(z-z_{k}^{ \pm}\right), \quad k=1, \ldots, N
$$

in the neighborhood of $z_{k}^{ \pm}$. The RHP (64) and the normalization condition (65) can be reformulated on the complex $z$-plane as follows

$$
\begin{aligned}
\xi^{+}(x, z) & =\xi^{-}(x, z) G(x, z), \quad \lim _{z \rightarrow \infty} \xi^{ \pm}(x, z)=\mathbb{1} \\
G(x, z) & =\mathrm{e}^{-\mathrm{i}\left(z-z^{-1}\right) \rho J x} \widehat{S}^{-} S^{+}(\lambda) \mathrm{e}^{\mathrm{i}\left(z-z^{-1}\right) \rho J x} .
\end{aligned}
$$




\subsection{Constructing Singular Solutions of the RHP}

The singularities of the FAS are directly related to the discrete eigenvalues of $L$. Of course we have to remember that the Lax operator $L$ is equivalent to a selfadjoint eigenvalue problem. Therefore its eigenvalues on the complex $\lambda$-plane must be real and lie in the lacuna, i.e., $-\rho<\lambda_{k}<\rho$. Going to the uniformizing variable we find that $\lambda_{k}$ is mapped on the unit circle of the complex $z$-plane

$$
z_{k}^{+}=\frac{\lambda_{k}+\mathrm{i} \sqrt{\rho^{2}-\lambda_{k}^{2}}}{2 \rho}, \quad z_{k}^{-}=\frac{1}{z_{k}^{+}}=\frac{\lambda_{k}-\mathrm{i} \sqrt{\rho^{2}-\lambda_{k}^{2}}}{2 \rho}
$$

so that $z_{k}^{ \pm} \in \mathbb{C}_{ \pm}$. Therefore it will be convenient to write down the anzats for the dressing factor as rational function of $z$ as follows

$$
u_{k}(x, z)=\mathbb{1}+\left(c_{k}(z)-1\right) P_{k}+\left(c_{k}^{-1}-1\right) \bar{P}_{k}, \quad c_{k}(z)=\frac{z-z_{k}^{+}}{z-z_{k}^{-}}
$$

where $P_{k}$ is a projector onto the corresponding eigen-subspace of $L$ and $\bar{P}_{k}=$ $S_{0} P_{k}^{T} S_{0}$. This last condition ensures that $u_{k}(x, z)$ is an element of the corresponding Lie group. It remains to introduce the asymptotic expansion of FAS over the negative powers of $z$

$$
\xi^{ \pm}(x, z)=\mathbb{1}+\sum_{k=1}^{\infty} z^{-k} \xi_{k}^{ \pm}(x)
$$

and to replace in equation (62) $\lambda$ and $\mu(\lambda)$ by $\rho\left(z+z^{-1}\right)$ and $\rho\left(z-z^{-1}\right)$ respectively. The corresponding equation

$$
\mathrm{i} \frac{\mathrm{d} \xi^{ \pm}}{\mathrm{d} x}+\left(Q(x)-\left(z+z^{-1}\right) \rho J\right) \xi^{ \pm}(x, z)+\xi^{ \pm}(x, z)\left(z-z^{-1}\right) \rho J=0
$$

allows one to relate the potential $Q(x)$ with the first coefficient $\xi_{1}^{ \pm}(x)$ in the expansion (71). Indeed, taking the limit $z \rightarrow \infty$ in equation (72) we get

$$
Q(x)=\lim _{z \rightarrow \infty} z \rho\left(J-\xi^{ \pm}(x, z) J \widehat{\xi}^{ \pm}(x, z)\right)=\left[J, \xi_{1}^{ \pm}(x)\right] .
$$

Let us assume that we have solved the RHP (68) and know one of its regular solutions $\xi_{0}^{ \pm}(x, z)$. Then equation (73) allows us to evaluate the corresponding regular potential $Q_{0}(x)$. Next we apply $N$ times the dressing procedure by acting with $u_{1}(x, z), \ldots, u_{N}(x, z)$, adding $2 N$ discrete eigenvalues $z_{k}^{ \pm}$to the spectrum 
of $L$. Then the resulting potential $Q(x)$ will be related to the regular one $Q_{0}(x)$ by

$$
\begin{aligned}
Q(x) & =Q_{0}(x)-\sum_{k=1}^{N}\left(z_{k}^{+}-z_{k}^{-}\right)\left[J, P_{k}-\bar{P}_{k}\right) \\
& =Q_{0}(x)-\frac{i}{\rho} \sum_{k=1}^{N} \sqrt{\rho^{2}-\lambda_{k}^{2}}\left[J, P_{k}-\bar{P}_{k}\right] .
\end{aligned}
$$

These expressions allow us to evaluate the $N$-soliton solutions of the MNLS equation with c.b.c. The explicit construction of the projectors $P_{k}(x)$ in terms of the regular solutions $\chi_{0}^{ \pm}(x, \lambda)$ will be presented elsewhere.

\subsection{The Resolvent of the Lax Operator}

The FAS can be used to construct the resolvent of the Lax operator, much in the same way as it has been done for the v.b.c. case. Let us define the kernel of the resolvent of $L$ by

$$
\begin{aligned}
R^{ \pm}(x, y, \lambda) & =\frac{1}{\mathrm{i}} \chi^{ \pm}(x, \lambda) \Theta^{ \pm}(x-y) \widehat{\chi}^{ \pm}(y, \lambda) \\
\Theta^{+}(x-y) & =\left(\begin{array}{cc}
-11 \theta(y-x) & 0 \\
0 & 11 \theta(x-y)
\end{array}\right) \\
\Theta^{-}(x-y) & =\left(\begin{array}{cc}
1 \theta(x-y) & 0 \\
0 & -11 \theta(y-x)
\end{array}\right), \quad \lambda \in C_{\rho}
\end{aligned}
$$

where $\chi^{ \pm}(x, \lambda)$ are given by equation (61).

Theorem 1. Let $Q(x)$ is a function such that $\lim _{x \rightarrow \pm \infty}\left(Q(x)-Q_{ \pm}\right)=0$ fast enough. Let $-\rho<\lambda_{j}<\rho$ be the simple zeroes of $\operatorname{det} \boldsymbol{a}^{ \pm}(\lambda)$. Then

1. $R^{ \pm}(x, y, \lambda)$ is an analytic function of $\lambda$ for $\lambda \in \mathcal{R}_{1,2}$ having pole singularities at $\lambda_{j}^{ \pm} \in[-\rho, \rho]$

2. $R^{ \pm}(x, y, \lambda)$ is a kernel of a bounded integral operator for $\operatorname{Im} \mu(\lambda) \neq 0$

3. $R^{ \pm}(x, y, \lambda)$ is uniformly bounded function for $\lambda \in \mathcal{R}_{1,2}$ and provides a kernel of an unbounded integral operator

4. $R^{ \pm}(x, y, \lambda)$ satisfy the equation

$$
L(\lambda) R^{ \pm}(x, y, \lambda)=\mathbb{1} \delta(x-y)
$$


Proof: Idea of the proof.

1. This is obvious from the fact that $\chi^{ \pm}(x, \lambda)$ are the FAS of $L(\lambda)$.

2. Assume that $\operatorname{Im} \mu(\lambda)>0$ and consider the asymptotic of $R^{+}(x, y, \lambda)$ for $x, y \rightarrow \infty$. From equations (27) and (63) we find that for $x, y \rightarrow-\infty$ the $(1,1)$-block of $\widehat{\psi}_{0} R^{+}(x, y, \lambda) \psi_{0}$ behaves like

$$
\left(\widehat{\psi}_{0}(\lambda) R^{+}(x, y, \lambda) \psi_{0}(\lambda)\right)_{11} \simeq \mathrm{ie}^{-\mathrm{i} \mu(\lambda)(x-y)} \theta(y-x)
$$

i.e., it decreases exponentially for all $x \rightarrow-\infty$ and arbitrary choice of $y$. Similar analysis applies also for the other blocks of $R^{+}(x, y, \lambda)$. All other possibilities are treated analogously. In doing this it is important that $\chi^{+}(x, \lambda)$ has triangular asymptotics for $x \rightarrow \pm \infty$ and also of the correct choice of $\Theta^{+}(x-y)$ (75). To conclude the proof we also use the fact that $\widehat{\psi}_{0}(\lambda)$ and $\psi_{0}(\lambda)$ are singular only at the end-points of the cut $C_{\rho}$.

3. For $\lambda \in C_{\rho}$ the arguments of 2) can not be applied because the exponentials in the right hand side of (77) $\operatorname{Im} \lambda=0$ only oscillate. Thus we conclude that $R^{ \pm}(x, y, \lambda)$ for $\lambda \in C_{\rho}$ is only a bounded function and thus the corresponding operator $R(\lambda)$ is an unbounded integral operator.

4. The proof of equation (76) follows from the fact that $L(\lambda) \chi^{+}(x, \lambda)=0$ and

$$
\frac{\mathrm{d} \Theta^{ \pm}(x-y)}{\mathrm{d} x}=\mp \mathbb{1} \delta(x-y) .
$$

The theorem is proved.

Using the kernel of the resolvent $R^{ \pm}(x, y, \lambda)$ one can derive the spectral decomposition of $L$.

To do this we apply the contour integration method to the integral

$$
\mathcal{J}_{R}(x, y)=\frac{1}{2 \pi \mathrm{i}} \int_{C} \frac{\lambda \mathrm{d} \lambda}{\mu(\lambda)} R^{+}(x, y, \lambda)
$$

where the contour is shown on the figure below.

According to Cauchy theorem

$$
\mathcal{J}_{R}(x, y)=\sum_{k=1}^{N} \operatorname{Res}_{\lambda=\lambda_{k}} \frac{\lambda}{\mu(\lambda)} R^{+}(x, y, \lambda) .
$$




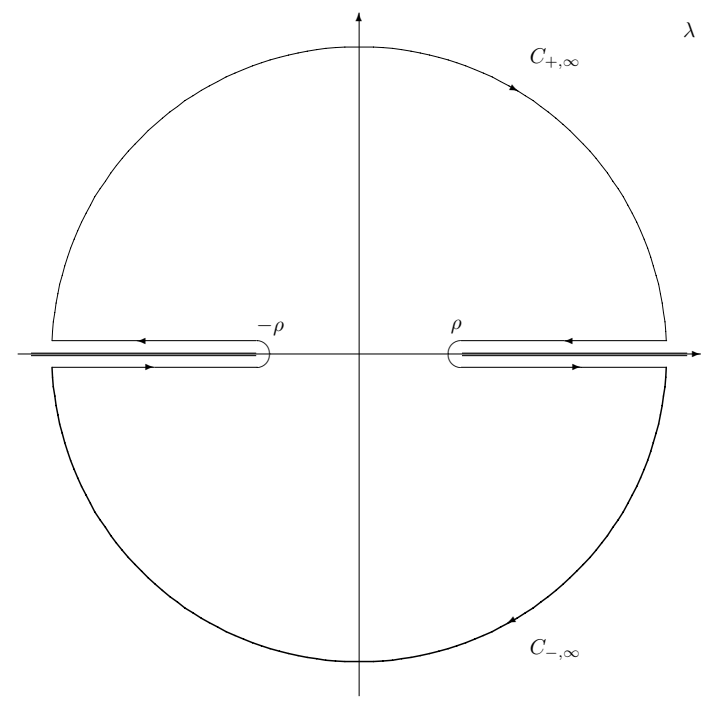

Figure 1. The contour along which we integrate lies completely on the first sheet of the 2-sheeted spectral surface associated with the square root $\mu(\lambda)=$ $\sqrt{\lambda^{2}-\rho^{2}}$

We can also calculate $\mathcal{J}_{R}$ by integrating along the contour. We split this integral into two and first evaluate the integral along $C_{\rho}$. Doing this we have to determine the jump of $R^{+}(x, y, \lambda)$ along the cut

$$
\begin{aligned}
& R^{+}(x, y, \lambda, \mu(\lambda))-R^{+}(x, y, \lambda,-\mu(\lambda)) \\
& \quad=R^{+}(x, y, \lambda, \mu(\lambda))-R^{-}(x, y, \lambda, \mu(\lambda)) \\
& \quad=\frac{1}{\mathrm{i}}\left(\left|\phi^{+}(x, \lambda)\right\rangle \widehat{\mathbf{a}}^{+}\left\langle\widehat{\psi}^{+}(y, \lambda)-\mid \phi^{-}(x, \lambda)\right\rangle \widehat{\mathbf{a}}^{-}\left\langle\widehat{\psi}^{-}(y, \lambda)\right) .\right.
\end{aligned}
$$

The integration along the infinite semi-arcs can be done explicitly using the asymptotic of $\chi^{+}(x, \lambda)$ for large $\lambda$. Note that the asymptotic is different for the upper and lower complex half-planes

$$
\begin{aligned}
\chi^{+}(x, \lambda)= & \psi_{0}(\lambda)\left(11+z^{-1} \xi_{1}^{+}(x)+\cdots\right) \mathrm{e}^{-\mathrm{i} \mu(\lambda) J x} \\
& \rightarrow \mathrm{e}^{-\mathrm{i} \lambda J x}\left(11+\mathcal{O}\left(\lambda^{-1}\right)\right), \quad \text { for } \lambda \in \mathbb{C}_{+} \\
\lambda & \rightarrow \infty \\
\lambda \rightarrow \infty & \mathrm{e}^{\mathrm{i} \lambda J x}\left(\mathrm{i} S+\mathcal{O}\left(\lambda^{-1}\right)\right), \quad \text { for } \lambda \in \mathbb{C}_{-}
\end{aligned}
$$

where

$$
S=\left(\begin{array}{cc}
0 & S_{1} \\
S_{1} & 0
\end{array}\right)
$$


As a result the integration over $C_{ \pm, \infty}$ can be performed explicitly with the result

$$
\frac{1}{2 \pi \mathrm{i}} \int_{C_{+, \infty} \cup C_{-, \infty}} \frac{\lambda \mathrm{d} \lambda}{\mu(\lambda)} R^{+}(x, y, \lambda)=\delta(x-y) J .
$$

It remains to equate the two answers for the integral $\mathcal{J}_{R}(x, y)$ in order to get the spectral decomposition for $L$ in the form

$$
\begin{aligned}
\delta(x-y) J & \\
= & -\frac{1}{2 \pi} \int_{C_{\rho}} \frac{\lambda \mathrm{d} \lambda}{\mu(\lambda)}\left(\left|\phi^{+}(x, \lambda)\right\rangle \widehat{\mathbf{a}}^{+}\left\langle\widehat{\psi}^{+}(y, \lambda)-\mid \phi^{-}(x, \lambda)\right\rangle \widehat{\mathbf{a}}^{-}\left\langle\widehat{\psi}^{-}(y, \lambda)\right)\right. \\
& +\sum_{k=1}^{N} \operatorname{Res}_{\lambda=\lambda_{k}} \frac{\lambda}{\mu(\lambda)} R^{+}(x, y, \lambda) .
\end{aligned}
$$

We recall that the $r \times 2 r$ blocks $\left|\phi^{ \pm}(x, \lambda)\right\rangle$ and $\left|\psi^{ \pm}(x, \lambda)\right\rangle$ are defined by equation (50) while the $r \times 2 r$ blocks $\left\langle\phi^{ \pm}(x, \lambda)\right|$ and $\left\langle\psi^{ \pm}(x, \lambda)\right|$ are introduced in equation (51).

In deriving equation (85) we have assumed in addition that $R^{ \pm}(x, y, \lambda)$ has no singularities at the end points of the spectrum.

\section{Wronskian Relations}

Let the class of allowed potentials $\mathcal{M}$ be a slice of $O_{J}$ determined by additional constraints: i) any generic element $F(x)=P_{J} F(x)$ of $\mathcal{M}$ is matrix-valued function such that $\lim _{x \rightarrow \pm \infty}\left(Q(x)-Q_{ \pm}\right)=0$ and ii) the phase factor $V=\exp \left(\mathrm{i} \varphi_{0} J\right)$ which connect the asymptotic values of the potential $Q_{+}=V^{\dagger} Q_{-} V$ and $\varphi_{0}$ is an integral of motion. The derivative of the potential $Q_{x}(x, t)$ belongs to the class of allowed potentials. The variation of the potential $\delta Q(x, t)$ is an allowed potential provided it satisfies the second additional condition. The mapping $\mathcal{F}: \mathcal{M} \rightarrow \mathcal{L}$ between the class of allowed potentials $\mathcal{M}$ and the scattering data $\mathcal{L}$ of $L$ is analyzed by means of Wronskian relations [5,6]. These relations allow us to formulate the main result of this work, i.e., that the ISM is a generalized Fourier transform in the case of C.I and D.III-type symmetric spaces. They also serve to introduce the skew-scalar product

$$
\llbracket A(x), B(x) \rrbracket=\frac{1}{2} \int \mathrm{d} x\langle A(x),[J, B(x)]\rangle
$$


which is non-degenerate for $A(x), B(x) \in \mathcal{M}$ and provides it with symplectic structure. We start with the identity

$$
\begin{aligned}
&\left.\left\langle\widehat{\chi}(Q(x, t)-\lambda J) \chi(x, \lambda), E_{ \pm \alpha}\right\rangle\right|_{x=-\infty} ^{\infty} \\
&=-\mathrm{i} \int_{-\infty}^{\infty} \mathrm{d} x\left\langle\frac{\mathrm{i}}{2}\left[J, J Q_{x}\right], P_{J} \chi E_{ \pm \alpha} \widehat{\chi}(x, \lambda)\right\rangle
\end{aligned}
$$

where $\chi(x, \lambda)$ can be any fundamental solution of $L$. For convenience we choose them to be the FAS introduced above. The 1.h.side of (87) can be calculated explicitly by using the asymptotics of FAS for $x \rightarrow \pm \infty$. It would be expressed by the matrix elements of the scattering matrix $T(\lambda)$, i.e., by the scattering data of $L$ as follows

$$
\begin{aligned}
{\left[\left[P_{J} \chi^{+}(x, \lambda) E_{\alpha} \widehat{\chi}^{+}, J Q_{x}\right]\right] } & =-\mu(\lambda)\left\langle\widehat{\mathbf{T}}^{-} J \mathbf{T}^{-}, E_{\alpha}\right\rangle=2 \mu(\lambda) \mathbf{b}_{\alpha}^{+} \\
{\left[\left[P_{J} \chi^{+}(x, \lambda) E_{-\alpha} \widehat{\chi}^{+}, J Q_{x}\right]\right] } & =\mu(\lambda)\left\langle\widehat{\mathbf{S}}^{+} J \mathbf{S}^{+}, E_{-\alpha}\right\rangle=2 \mu(\lambda) \mathbf{d}_{-\alpha}^{-} \\
{\left[\left[P_{J} \chi^{-}(x, \lambda) E_{\alpha} \widehat{\chi}^{-}, J Q_{x}\right]\right] } & =\mu(\lambda)\left\langle\widehat{\mathbf{S}}^{-} J \mathbf{S}^{-}, E_{\alpha}\right\rangle=2 \mu(\lambda) \mathbf{d}_{\alpha}^{+} \\
{\left[\left[P_{J} \chi^{-}(x, \lambda) E_{-\alpha} \widehat{\chi}^{-}, J Q_{x}\right]\right] } & =-\mu(\lambda)\left\langle\widehat{\mathbf{T}}^{+} J \mathbf{T}^{+}, E_{-\alpha}\right\rangle=2 \mu(\lambda) \mathbf{b}_{-\alpha}^{-}
\end{aligned}
$$

where $\alpha \in \triangle_{1}^{+}$.

The second set of Wronskian relations which we consider relate the variation of the potential $\delta Q$ to the corresponding variations of the scattering data $\delta \rho$ and $\delta \tau$. For this purpose we use the identity

$$
\left.\left\langle\widehat{\chi} \delta \chi(x, \lambda), E_{ \pm \alpha}\right\rangle\right|_{x=-\infty} ^{\infty}=\frac{\mathrm{i}}{2} \int_{-\infty}^{\infty} \mathrm{d} x\left\langle[J, J \delta Q], P_{J} \chi(x, \lambda) E_{ \pm \alpha} \widehat{\chi}\right\rangle .
$$

If we assume that the variation of the phase factor $\delta V$ vanishes we arrive at

$$
\begin{aligned}
{\left[\left[P_{J} \chi^{+}(x, \lambda) E_{\alpha} \widehat{\chi}^{+}, J \delta Q\right]\right] } & =-\mathrm{i}\left\langle\widehat{\mathbf{T}}^{-} \delta \mathbf{T}^{-}, E_{\alpha}\right\rangle=\mathrm{i}\left(\delta \rho^{+} \mathbf{a}^{+}\right)_{\alpha} \\
{\left[\left[P_{J} \chi^{+}(x, \lambda) E_{-\alpha} \widehat{\chi}^{+}, J \delta Q\right]\right] } & =\mathrm{i}\left\langle\widehat{\mathbf{S}}^{+} \delta \mathbf{S}^{+}, E_{-\alpha}\right\rangle=\mathrm{i}\left(\delta \tau^{+} \mathbf{c}^{+}\right)_{-\alpha} \\
{\left[\left[P_{J} \chi^{-}(x, \lambda) E_{\alpha} \widehat{\chi}^{-}, J \delta Q\right]\right] } & =\mathrm{i}\left\langle\widehat{\mathbf{S}}^{-} \delta \mathbf{S}^{-}, E_{\alpha}\right\rangle=\mathrm{i}\left(\delta \tau^{-} \mathbf{c}^{-}\right)_{\alpha} \\
{\left[\left[P_{J} \chi^{-}(x, \lambda) E_{-\alpha} \widehat{\chi}^{-}, J \delta Q\right]\right] } & =-\mathrm{i}\left\langle\widehat{\mathbf{T}}^{+} \delta \mathbf{T}^{+}, E_{-\alpha}\right\rangle=\mathrm{i}\left(\delta \rho^{-} \mathbf{a}^{-}\right)_{-\alpha}
\end{aligned}
$$

where $\alpha \in \triangle_{1}^{+}$.

These relations are basic for the analysis of the related NLEE and their Hamiltonian structures. The above identities also allow us to introduce the proper generalizations of the usual Fourier exponential functions. Let us introduce the set of 
"squared solutions"

$$
\begin{aligned}
& \Phi_{\alpha}^{ \pm}(x, \lambda)=P_{J} \chi^{ \pm}(x, \lambda) E_{ \pm \alpha} \widehat{\chi}^{ \pm}(x, \lambda), \quad \text { for } \alpha \in \Delta_{1}^{+} \\
& \Psi_{\alpha}^{ \pm}(x, \lambda)=P_{J} \chi^{ \pm}(x, \lambda) E_{\mp \alpha} \widehat{\chi}^{ \pm}(x, \lambda), \quad \text { for } \alpha \in \Delta_{1}^{+} \\
& \Theta_{\alpha}^{ \pm}(x, \lambda)=P_{J} \chi^{ \pm}(x, \lambda) E_{ \pm \alpha} \widehat{\chi}^{ \pm}(x, \lambda), \quad \text { for } \alpha \in \Delta_{0}^{+} \\
& \Xi_{\alpha}^{ \pm}(x, \lambda)=P_{J} \chi^{ \pm}(x, \lambda) E_{\mp \alpha} \widehat{\chi}^{ \pm}(x, \lambda), \quad \text { for } \alpha \in \Delta_{0}^{+} \\
& \Upsilon_{k}^{ \pm}(x, \lambda)=P_{J} \chi^{ \pm}(x, \lambda) H_{k} \widehat{\chi}^{ \pm}(x, \lambda), \quad \text { for } k=1, \ldots, r \text {. }
\end{aligned}
$$

These are the "squared solutions" of the Lax operator $L$ connected with simple Lie algebra $\mathfrak{g}$. They are constructed by means of FAS $\chi^{ \pm}(x, \lambda)$ and the CartanWeyl basis of the algebra and are analytic functions of $\lambda$ on the corresponding sheets of the spectral surface. The equations that $\Phi_{\alpha}^{ \pm}$an $\Psi_{\alpha}^{ \pm}$satisfy are a direct consequence of the fact that FAS and their inverse satisfy the Z-Sh system system $\mathrm{i} \frac{\mathrm{d} \Phi_{\alpha}^{ \pm}}{\mathrm{d} x}+\left[Q(x)-\lambda J, \Phi_{\alpha}^{ \pm}(x, \lambda)\right]=0, \quad \mathrm{i} \frac{\mathrm{d} \Psi_{\alpha}^{ \pm}}{\mathrm{d} x}+\left[Q(x)-\lambda J, \Psi_{\alpha}^{ \pm}(x, \lambda)\right]=0$.

The "squared solutions" serve as building blocks of the Green function [8-10]

$$
\mathbf{G}^{ \pm}(x, y, \lambda)=G_{1}^{ \pm}(x, y, \lambda) \theta(y-x)-G_{2}^{ \pm}(x, y, \lambda) \theta(x-y)
$$

which allows one to derive their completeness relation. Here

$$
\begin{aligned}
G_{1}^{ \pm}(x, y, \lambda)= & \sum_{\alpha \in \triangle_{1}^{+}} \Phi_{\alpha}^{ \pm}(x, \lambda) \otimes \Psi_{\alpha}^{ \pm}(y, \lambda) \\
G_{2}^{ \pm}(x, y, \lambda)= & \sum_{\alpha \in \triangle_{1}^{+}} \Psi_{\alpha}^{ \pm}(x, \lambda) \otimes \Phi_{\alpha}^{ \pm}(y, \lambda)+\sum_{\alpha \in \triangle_{0}^{+} \cup \triangle_{1}^{+}} \Xi_{\alpha}^{ \pm}(x, \lambda) \otimes \Theta_{\alpha}^{ \pm}(y, \lambda) \\
& +\sum_{k=1}^{r} \Upsilon_{k}^{ \pm}(x, \lambda) \otimes \Upsilon_{k}^{ \pm}(y, \lambda)
\end{aligned}
$$

and $\theta(x)$ is the usual step function.

\section{Generalized Fourier Transforms and Higher MNLS}

The main result in this section is that the sets $\left\{\Phi_{\alpha}^{ \pm}\right\}$and $\left\{\Psi_{\alpha}^{ \pm}\right\}$form complete sets of functions in $\mathcal{M}$. The idea of the proof is simple. Apply the contour integration method along a proper contour (see Fig.1) to a conveniently chosen Green function (92). From the Cauchy theorem we have

$$
\frac{1}{2 \pi \mathrm{i}} \oint_{C} \frac{\lambda \mathrm{d} \lambda}{\mu(\lambda)} \mathbf{G}^{+}(x, y, \lambda)=\sum_{k=1}^{N} \operatorname{Res}_{\lambda=\lambda_{k}^{ \pm}} \frac{\lambda}{\mu(\lambda)} \mathbf{G}^{+}(x, y, \lambda) .
$$


Integrating along the contours we treat separately the contribution from the infinite semi-arcs and the ones from the continuous spectrum $R_{m}=C_{1} \cup C_{2}$ which is composed of the cuts $C_{1}=(-\infty,-m)$ and $C_{2}=(m, \infty)$. Special care must be taken for the end points $\lambda= \pm m$ of the spectrum. Assuming that the end points of the spectrum give no contribution we obtain the following completeness relation

$$
\begin{aligned}
& \delta(x-y) \Pi_{J} \\
& =\frac{1}{\pi} \sum_{\alpha \in \triangle_{1}^{+}} \int_{R_{m}} \frac{\lambda \mathrm{d} \lambda}{\mu(\lambda)}\left\{\Phi_{\alpha}^{+}(x, \lambda) \otimes \Psi_{\alpha}^{+}(y, \lambda)-\Phi_{\alpha}^{-}(x, \lambda) \otimes \Psi_{\alpha}^{-}(y, \lambda)\right\} \\
& -2 \mathrm{i} \sum_{\alpha \in \triangle_{1}^{+}} \sum_{k=1}^{N}\left(\frac{\mathrm{d}}{\mathrm{d} \lambda} \frac{\lambda}{\mu(\lambda)} \Phi_{\alpha ; k}^{+}(x) \otimes \Psi_{\alpha ; k}^{+}(y)\right)_{\left.\right|_{\lambda=\lambda_{k}} .}
\end{aligned}
$$

Here $\Pi_{J}=\sum_{\alpha \in \triangle_{1}^{+}}\left[E_{\alpha} \otimes E_{-\alpha}-E_{-\alpha} \otimes E_{\alpha}\right]$. The assumption that we have made is that $\lambda_{j}^{+}$are simple poles of the "squared solutions" $\Phi_{\alpha}^{+}$and $\Psi_{\alpha}^{+}$.

Using the completeness relation one can expand any generic element of the phase space $\mathcal{M}$ over each of the complete sets of "squared solutions" $\Psi_{\alpha}^{ \pm}$and $\Phi_{\alpha}^{ \pm}$. This relation is utilized with the help of the following the trick

$$
-\frac{1}{2} \operatorname{tr}_{1}\left\{([J, F(x)] \otimes \mathbb{1}) \Pi_{J}\right\}=\frac{1}{2} \operatorname{tr}_{2}\left\{\Pi_{J}(\mathbb{1} \otimes[J, F(x)])\right\}=F(x)
$$

where $\operatorname{tr}_{1}$ (and $\operatorname{tr}_{2}$ ) mean taking the trace of the elements in the first (or in the second) position of the tensor product.

The completeness relation (96) allows to establish one-to-one correspondence between the elements of $\mathcal{M}$, such as $Q_{x}$ and $Q_{t}$, and its expansion coefficients. It is also directly related to the spectral decompositions of the generating (recursion) operators $\Lambda_{ \pm}$. These operators are the ones whose eigenfunctions are the "squared solutions" [8]. For an alternative method of constructing recursion operators see [17]. There the derivation starts by introducing the splitting of the object $e_{\alpha}^{ \pm}=\chi^{ \pm}(x, \lambda) E_{ \pm \alpha} \widehat{\chi}^{ \pm}(x, \lambda)$ into block diagonal and block off-diagonal parts

$$
e_{\alpha}^{ \pm}(x, \lambda)=e_{\alpha}^{d, \pm}(x, \lambda)+\Phi_{\alpha}^{ \pm}(x, \lambda), \quad e_{\alpha}^{d, \pm}(x, \lambda)=\left(\mathbb{1}-P_{J}\right) e_{\alpha}^{ \pm}(x, \lambda)
$$

and making use of the equation

$$
\mathrm{i} \frac{\mathrm{d} e_{\alpha}^{ \pm}}{\mathrm{d} x}+\left[Q(x)-\lambda J, e_{\alpha}^{ \pm}(x, \lambda)\right]=0 .
$$

The last equation splits into

$$
\mathrm{i} \frac{\mathrm{d} e_{\alpha}^{d, \pm}}{\mathrm{d} x}+\left[Q(x), \Phi_{\alpha}^{ \pm}(x, \lambda)\right]=0
$$


and

$$
\mathrm{i} \frac{\mathrm{d} \Phi_{\alpha}^{ \pm}}{\mathrm{d} x}+\left[Q(x), e_{\alpha}^{d, \pm}(x, \lambda)\right]=\lambda\left[J, \Phi_{\alpha}^{ \pm}(x, \lambda)\right] .
$$

Further, equation (100) can be integrated formally which gives

$$
\begin{gathered}
e_{\alpha}^{d, \pm}(x, \lambda)=C_{\alpha ; \epsilon}^{d, \pm}(\lambda)+\mathrm{i} \int_{\epsilon \infty}^{x} \mathrm{~d} y\left[Q(y), \Phi_{\alpha}^{ \pm}(y, \lambda)\right] \\
C_{\alpha ; \epsilon}^{d, \pm}(\lambda)=\lim _{x \rightarrow \epsilon \infty} e_{\alpha}^{d, \pm}(x, \lambda), \quad \epsilon= \pm 1 .
\end{gathered}
$$

Next insert (102) into (101) and act on both sides by $\operatorname{ad}_{J}^{-1}$. This gives us

$$
\left(\Lambda_{ \pm}-\lambda\right) \Phi_{\alpha}^{ \pm}(x, \lambda)=\mathrm{i}\left[C_{\alpha ; \epsilon}^{d, \pm}(\lambda), \operatorname{ad}_{J}^{-1} Q(x)\right]
$$

where the generating operators $\Lambda_{ \pm}$are given by

$$
\Lambda_{ \pm} \Xi(x)=\operatorname{ad}_{J}^{-1}\left\{\mathrm{i} \frac{\mathrm{d} \Xi}{\mathrm{d} x}+\mathrm{i}\left[Q(x), \int_{ \pm \infty}^{x} \mathrm{~d} y[Q(y), \Xi(y)]\right]\right\} .
$$

Thus $\Psi_{\alpha}^{ \pm}$(respectively $\Phi_{\alpha}^{ \pm}$) will be eigenfunctions of $\Lambda_{+}$(respectively $\Lambda_{-}$) if and only if $C_{\alpha ; \epsilon}^{d, \pm}(\lambda)=0$. Evaluating the limit of (103) for all $\alpha$ we find

$$
\begin{aligned}
& \left(\Lambda_{+}-\lambda\right) \Psi_{\alpha}^{ \pm}(x, \lambda)=0, \quad\left(\Lambda_{+}-\lambda_{j}^{ \pm}\right) \Psi_{\alpha, j}^{ \pm}(x)=0 \\
& \left(\Lambda_{-}-\lambda\right) \Phi_{\alpha}^{ \pm}(x, \lambda)=0, \quad\left(\Lambda_{-}-\lambda_{j}^{ \pm}\right) \Phi_{\alpha, j}^{ \pm}(x)=0
\end{aligned}
$$

where $\alpha \in \triangle_{1}^{+}$. This result can be generalized for arbitrary $f\left(\Lambda_{ \pm}\right)$

$$
\begin{array}{ll}
\left(f\left(\Lambda_{+}\right)-f(\lambda)\right) \Psi_{\alpha}^{ \pm}(x, \lambda)=0, & \left(f\left(\Lambda_{+}\right)-f\left(\lambda_{j}^{ \pm}\right)\right) \Psi_{\alpha ; j}^{ \pm}(x)=0 \\
\left(f\left(\Lambda_{-}\right)-f(\lambda)\right) \Phi_{\alpha}^{ \pm}(x, \lambda)=0, & \left(f\left(\Lambda_{-}\right)-f\left(\lambda_{j}^{ \pm}\right)\right) \Phi_{\alpha, j}^{ \pm}(x)=0 .
\end{array}
$$

The class of higher MNLS on symmetric spaces of C.I and D.III-type and with c.b.c. can be put down in terms of the derivative of the potential $Q_{t}$ with respect to the evolution parameter and the dispersion law $-2 \mu(\lambda) f(\lambda)[10,25]$ as follows

$$
\operatorname{iad}_{J}^{-1} \frac{\partial}{\partial t} Q+f(\Lambda) \operatorname{ad}_{J}^{-1} Q_{x}=0
$$

Substituting the objects in this formula with their expansions over the "squared solutions" we obtain equations for the evolution of the scattering data. The expansion coefficients of $\operatorname{ad}_{J}^{-1} Q_{t}$ and $\operatorname{ad}_{J}^{-1} Q_{x}$ on the continuous spectrum turn out 
to be exactly the minimal set of scattering data. The evolution for the reflection and transition coefficients is provided by

$$
\mathrm{i} \frac{\mathrm{d} \rho^{ \pm}}{\mathrm{d} t} \mp f(\lambda) \mu(\lambda) \rho^{ \pm}(t, \lambda)=0, \quad \mathrm{i} \frac{\mathrm{d} \tau^{ \pm}}{\mathrm{d} t} \pm f(\lambda) \mu(\lambda) \tau^{ \pm}(t, \lambda)=0
$$

for $\lambda \in R_{m}$.

The observation that the scattering data evolves trivially is visible from the equation depicting the evolution of the scattering matrix $T(\lambda)$. This equation is a result of the compatibility condition (8) and the fact that the two Jost solutions $\psi$ and $\phi$ are solutions of the second operator of the Lax pair in the Z-Sh system (9). Acting with id/d $t$ on $T(\lambda)$ (31), we recover equation (35).

We end this Section by the principal series of local integrals of motion $I_{k}$ which is generated by:

$$
\ln \operatorname{det} \mathbf{a}^{ \pm}(\lambda)=\sum_{k=1}^{\infty} \lambda^{-k} I_{k}
$$

\section{Discussions and Conclusions}

Here we first briefly consider a more difficult problem: the spectral properties of the Lax operator $L$ with multiple thresholds. In order to deal with it we need to evaluate the asymptotic behavior of the Jost solutions for $x \rightarrow \pm \infty$. To this end we need to calculate the eigenvalues and eigenvectors of $Q_{ \pm}-\lambda J$

$$
\begin{aligned}
& \left(Q_{+}-\lambda J\right) \psi_{0}(\lambda)=-\psi_{0}(\lambda) J_{0}(\lambda) \\
& \left(Q_{-}-\lambda J\right) \phi_{0}(\lambda)=-\phi_{0}(\lambda) J_{0}(\lambda)
\end{aligned}
$$

which have the form

$$
\begin{aligned}
\psi_{0}(\lambda) & =\varphi_{0}^{+} U_{0}(\lambda), \quad \phi_{0}(\lambda)=\varphi_{0}^{-} U_{0}(\lambda), \quad \varphi_{0}^{ \pm}=\left(\begin{array}{cc}
\underline{\varphi}_{1}^{ \pm} & 0 \\
0 & \underline{\varphi}_{2}^{ \pm}
\end{array}\right) \\
U_{0}(\lambda) & =\sum_{k=1}^{r}\left(A_{k}\left(E_{k, k}+E_{\bar{k}, \bar{k}}\right)+B_{k}\left(E_{k, \bar{k}}+E_{\bar{k}, k}\right)\right) \\
A_{k} & =\sqrt{\frac{\lambda+j_{k}}{2 j_{k}}}, \quad B_{k}=\sqrt{\frac{\lambda-j_{k}}{2 j_{k}}}, \quad \bar{k}=2 r+1-k \\
q_{ \pm} q_{ \pm}^{\dagger} \underline{\varphi}_{1}^{ \pm} & =\underline{\varphi}_{1}^{ \pm} \underline{\rho}^{2}, \quad q_{ \pm}^{\dagger} q_{ \pm} \underline{\varphi}_{2}^{ \pm}=\underline{\varphi}_{2}^{ \pm} \underline{\rho}^{2}
\end{aligned}
$$

where $\rho=\operatorname{diag}\left(\rho_{1}, \ldots, \rho_{r}\right)$ is the set of threshold values. In what follows we assume that they are ordered by $\rho_{1}>\rho_{2}>\cdots>\rho_{r}>0$. We shall see that these eigenvalues determine the thresholds of continuous spectrum of $L$. 
The first difficulty in solving the problem is that the multiplicity of the continuous spectrum varies, which reflects on the definition of the Jost solutions

$$
\begin{array}{rlrl}
\psi(x, \lambda) \underset{x \rightarrow \infty}{\longrightarrow} \psi_{0}(\lambda) \mathrm{e}^{-\mathrm{i} J x} P(\lambda), & \phi(x, \lambda) \underset{x \rightarrow-\infty}{\longrightarrow} \phi_{0}(\lambda) \mathrm{e}^{-\mathrm{i} J x} P(\lambda) \\
\mu(\lambda) & =\sum_{k=1}^{r} \mu_{k}(\lambda) H_{k}, & P(\lambda) & =\sum_{k=1}^{r} P_{k}(\lambda)\left(E_{k, k}+E_{\bar{k}, \bar{k}}\right) \\
\mu_{k}(\lambda) & =\sqrt{\lambda^{2}-\rho_{k}^{2}}, & P_{k}(\lambda) & =\theta\left(|\operatorname{Re} \lambda|-\rho_{k}\right) .
\end{array}
$$

where $\bar{k}=2 r+1-k$.

We introduce $X^{ \pm}(x, \lambda)=\psi_{0}^{-1} \psi(x, \lambda) \exp \left(\mathrm{i} J_{0}(\lambda) x\right)$ which satisfy the following integral equations

$$
\begin{gathered}
X^{+}(x, \lambda)=\mathbb{1}+\mathrm{i} \int_{\infty}^{x} \mathrm{~d} y \mathrm{e}^{\mathrm{i} J_{0}(\lambda)(y-x)} U_{+}(x, \lambda) X^{+}(y, \lambda) \mathrm{e}^{-\mathrm{i} J_{0}(\lambda)(y-x)} \\
X^{-}(x, \lambda)=\mathbb{1}+\mathrm{i} \int_{-\infty}^{x} \mathrm{~d} y \mathrm{e}^{\mathrm{i} J_{0}(\lambda)(y-x)} U_{-}(x, \lambda) X^{-}(y, \lambda) \mathrm{e}^{-\mathrm{i} J_{0}(\lambda)(y-x)}
\end{gathered}
$$

or in components

$$
\begin{gathered}
X_{k l}^{+}(x, \lambda)=\delta_{k l}+\mathrm{i} \int_{\infty}^{x} \mathrm{~d} y \mathrm{e}^{\mathrm{i}\left(\mu_{k}(\lambda)-\mu_{k}(\lambda)\right)(y-x)}\left(U_{+}(x, \lambda) X^{+}(y, \lambda)\right)_{k l} \\
X_{k l}^{-}(x, \lambda)=\delta_{k l}+\mathrm{i} \int_{=\infty}^{x} \mathrm{~d} y \mathrm{e}^{\mathrm{i}\left(\mu_{k}(\lambda)-\mu_{k}(\lambda)\right)(y-x)}\left(U_{-}(x, \lambda) X^{-}(y, \lambda)\right)_{k l} .
\end{gathered}
$$

The second difficulty is that with each thresholds value $\rho_{k}$ one relates a Riemannian surface $\mathcal{S}^{(k)}, k=1, \ldots, r$ with two leafs defined by the sign of $\operatorname{Im} \mu_{k}(\lambda)$, thus we have a total of $2^{r}$ leafs. Below we define $\mathcal{R}_{1}$ by

$$
\operatorname{Im} \mu_{1}(\lambda)>\operatorname{Im} \mu_{2}(\lambda)>\cdots>\operatorname{Im} \mu_{r}(\lambda)>0
$$

and $\overline{\mathcal{R}}_{1}$ by

$$
\operatorname{Im} \mu_{1}(\lambda)<\operatorname{Im} \mu_{2}(\lambda)<\cdots<\operatorname{Im} \mu_{r}(\lambda)<0 .
$$

Now only the first column of $\psi(x, \lambda)$ and the last column of $\phi(x, \lambda)$ (respectively the last column of $\psi(x, \lambda)$ and the first column of $\phi(x, \lambda))$ have analyticity properties in $\lambda$ on $\mathcal{R}_{1}$ (respectively on $\overline{\mathcal{R}}_{1}$ ). Nevertheless using the methods proposed in [24] we will be able to construct fundamental analytic solutions as follows

$$
\begin{aligned}
& \chi^{+}(x, \lambda)=\psi(x, \lambda) T^{-} D^{+}(\lambda)=\phi(x, \lambda) S^{+}(\lambda) \\
& \chi^{-}(x, \lambda)=\psi(x, \lambda) T^{+} D^{-}(\lambda)=\phi(x, \lambda) S^{-}(\lambda)
\end{aligned}
$$


and show that $\chi^{+}(x, \lambda)$ (respectively $\left.\chi^{-}(x, \lambda)\right)$ is analytic ${ }^{1}$ in $\lambda$ on the sheet $\Re_{1}$ (respectively $\bar{\Re}_{1}$ ). In (120) $S^{+}, T^{+}$(respectively $S^{-}, T^{-}$) are the upper(respectively the lower-) triangular matrices, related to the scattering matrix $T(\lambda)$ by the Gauss factorization condition

$$
T(\lambda)=T^{-} D^{+}(\lambda) S^{+}(\lambda)=T^{+} D^{-} S^{+}(\lambda) .
$$

More detailed analysis, including the the proof of the above facts will be given elsewhere.

The result of this work is that the interpretation of the ISM as a generalized Fourier transformation holds true in the case of Lax operators with constant boundary conditions on symmetric spaces connected with the Lie algebras $C_{r} \simeq \mathfrak{s p}(2 r)$ and $D_{r} \simeq \mathfrak{s o}(2 r)$. The completeness relation of the "squared solutions" of the generalized Z-Sh system in the case when the Lax operator $L$ becomes self-adjoint is derived. The "squared solutions" turn out to be generalizations of the usual Fourier exponential function and eigenfunctions of the recursion operators $\Lambda_{ \pm}$. This result allows one to prove that the corresponding NLEE results in linear evolution for the scattering data. The recursion operators $[8,17] \Lambda_{ \pm}$open the path towards the construction of action-angle variables for the NLEE solvable with this generalization of the Z-Sh system and from there the Hamiltonian formulation of these equations and their hierarchies connected with $\Lambda_{ \pm}$. Similar constructions can be developed also for systems with deep reductions, see [26].

The physical applications of the NLS equation both with vanishing and nonvanishing boundary conditions is well known, the same holds true for the Manakov system as well as for the $\mathfrak{s p}(4)$ MNLS with v.b.c., see [19]. It will be interesting to find new physical applications also for the MNLS with c.b.c.

\section{Acknowledgements}

We thank Dr. V. Atanasov and Dr. T. Valchev for useful discussions.

\section{Appendix}

The above definition of $\mathfrak{g}$ (23) satisfies the requirement that the Cartan subalgebra $\mathfrak{h}$ will be made up of diagonal matrices. The Cartan generators $H_{k}$, dual to $e_{k}$, are

\footnotetext{
${ }^{1}$ More precisely, analytic functions in $\lambda$ are not $\chi^{ \pm}$and $\chi^{ \pm}$, but $\chi^{ \pm} \exp (\mathrm{i} J x)$ and $\chi^{ \pm} \exp (\mathrm{i} J x)$ respectively.
} 
given by

$$
H_{k}=E_{k k}-E_{\bar{k} \bar{k}}
$$

The element $J=\sum_{k=1}^{r} H_{k}$, belongs to $\mathfrak{h}$ and is dual to $\vec{a}$.

The root vectors in the typical representation are given by

$$
E_{e_{i}-e_{j}}=E_{i j}-(-1)^{i+j} E_{\bar{j} \bar{i}}, \quad E_{e_{i}+e_{j}}=E_{i \bar{j}}-\epsilon_{0}(-1)^{i+j} E_{j \bar{i}}
$$

where $1 \leq i<j \leq r$ and $\epsilon_{0}= \pm 1$. Since $\epsilon_{0}=1$ for $\mathfrak{g} \simeq \mathfrak{s o}(2 r)$ equation (123) gives vanishing result for $i=j$ which is compatible with the fact that $2 e_{i}$ are not roots of $\mathfrak{s o}(2 r)$; for $\mathfrak{g} \simeq \mathfrak{s p}(2 r) \epsilon_{0}=-1$ and equation (123) by putting $i=j$ provides also an expression for $E_{2 e_{i}}$. However this expression is not normed with respect to the Killing form $\left\langle E_{\alpha}, E_{-\alpha}\right\rangle=2$. The Weyl generators associated with the root $2 e_{i}$ that we will use are given by [18]

$$
E_{2 e_{i}}=\sqrt{2} E_{i \bar{i}} .
$$

\section{References}

[1] Ablowitz M., Kaup D., Newell A. and Segur H., The Inverse Scattering Transform - Fourier Analysis for Nonlinear Problems, Stud. Appl. Math 53 (1974) 249-315.

[2] Ablowitz M., Prinari B. and Trubatch A., Discrete and Continuous Nonlinear Schrödinger Systems, Cambridge Univ. Press, Cambridge, 2004.

[3] Ablowitz M. and Segur H., Solitons and the Inverse Scattering Transform, SIAM Studies in Applied Mathematics, SIAM, Philadelphia, 1981.

[4] Atanasov V. and Gerdjikov V., On the Multi-component Nonlinear Schrödinger Equation with Constant Boundary Conditions, In: Gravity, Astrophysics and Strings'05, P. Fiziev and M. Todorov (Eds), St. Kliment Ohridsky University Press, Sofia 2006, pp 22-36.

[5] Calogero F. and Degasperis A., Nonlinear Evolution Equations Solvable by the Inverse Spectral Transform I, Nuovo Cim. B 32 (1976) 201-242.

[6] Calogero F. and Degasperis A., Nonlinear Evolution Equations Solvable by the Inverse Spectral Transform II, Nuovo Cim. B 39 (1976) 1-54.

[7] Fordy A. and Kulish P., Nonlinear Schrödinger Equations and Simple Lie Algebras, Commun. Math. Phys. 89 (1983) 427-443.

[8] Gerdjikov V., Generalised Fourier Transforms for the Soliton Equations. Gauge Covariant Formulation, Inv. Problems 2 (1986) 51-74. 
[9] Gerdjikov V., Generating Operators for the Nonlinear Evolution Equations of Soliton Type Related to the Semisimple Lie Algebras (in Russian), Doctor of Sciences Thesis, JINR, Dubna, USSR, 1987.

[10] Gerdjikov V., Basic Aspects of Soliton Theory, In: Geometry, Integrability and Quantization VI, I. Mladenov and A. Hirshfeld (Eds), SOFTEX, Sofia 2005, pp. 78-125.

[11] Gerdjikov V., Selected Aspects of Soliton Theory. Constant Boundary Conditions, In: Prof. G. Manev's Legacy in Contemporary Aspects of Astronomy, Gravitational and Theoretical Physics, V. Gerdjikov and M. Tsvetkov (Eds), Heron Press, Sofia 2005, pp 277-290.

[12] Gerdjikov V., On Spectral Theory of Lax Operators on Symmetric Spaces: Vanishing Versus Constant Boundary Conditions, JGSP 15 (2009) 1-41.

[13] Gerdjikov V., Grahovski G. and Kostov N., Reductions of N-wave Interactions Related to Low-Rank Simple Lie Algebras: I. $\mathbb{Z}_{2}$-reductions, J. Phys. A 34 (2001) 9425-9461.

[14] Gerdjikov V., Kostov N. and Valchev T., On Multi-component NLS Equations with Constant Boundary Conditions, Theor. Math. Phys. 159 (2009) 786-794.

[15] Gerdjikov V. and Kulish P., On the Multicimponent Nonlinear Schrödinger Equation in the Case of Non-vanishing Boundary Conditions, Sci. Notes of LOMI Seminars 131 (1983) 34-46.

[16] Gerdjikov V., Vilasi G. and Yanovski A., Integrable Hamiltonian Hierarchies. Spectral and Geometric Methods, Lect. Notes in Physics 748, Springer, Berlin, 2008.

[17] Gürses M., Karasu A. and Sokolov V., On Construction of Recursion Operators From Lax Representation, J. Math. Phys. 40 (1999) 6473; doi:10.1063/1.533102 (18 pages)

[18] Helgasson S., Differential Geometry, Lie Groups and Symmetric Spaces, Academic Press, New York, 1978.

[19] Ieda J., Miyakawa T. and Wadati M., Exact Analysis of Soliton Dynamics in Spinor Bose-Einstein Condensates, Phys. Rev. Lett. 93 (2004) 194102.

[20] Kaup D., Closure of the Squared Zakharov-Shabat Eigenstates, Math. Annal. Appl. 54 (1976) 849-864.

[21] Konotop V. and Vekslerchik V., Direct Perturbation Theory for Dark Solitons, Phys. Rev. E 49 (1994) 2397-2407.

[22] Manakov S., On the Theory of Two-dimentional Stationary Self-focusing of Electromagnetic Waves, Sov. Phys. JETF 38 (1974) 248-253. 
[23] Mikhailov A., The Reduction Problem and the Inverse Scattering Problem, Physica D 3 (1981) 73-117.

[24] Shabat A., The Inverse Scattering Problem, Diff. Eqs. 15 (1979) 1824-1834.

[25] Takhtadjan L. and Faddeev L., Hamiltonian Approach to Soliton Theory, Springer, Berlin, 1986.

[26] Valchev T., On Generalized Fourier Transform for Kaup-Kupershmidt Type Equations, JGSP 19 (2010) 73-86.

[27] Zakharov V., Manakov S., On the Complete Integrability of the Nonlinear Schrödinger Equation (In Russian), TMP 19 (1974) 332-343.

[28] Zakharov V., Manakov S., Novikov S. and Pitaevskii L., The Theory of Solitons. The Inverse Transform Method (in Russian), Nauka, Moscow, 1980.

[29] Zakharov V. and Shabat A., Exact Theory of Two-dimentional Self-focusing and One-dimentional Modulation of Waves in Nonlinear Media (in Russian), Sov. Phys JETF 34 (1972) 62-69.

[30] Zakharov V. and Shabat A., On the Interaction of Solitons in Stable Medium (in Russian), Sov. Phys. JETF 37 (1973) 823-828.

[31] Zakharov V. and Shabat A., Integration of Nonlinear Equations of Mathematical Physics by the Inverse Scattering Method. I., Funct. Annal. Appl. 8 (1974) 43-53.

[32] Zakharov V. and Shabat A., Integration of Nonlinear Equations of Mathematical Physics by the Inverse Scattering Method. II, Funct. Anal. Appl. 13 (1979) 13-23.

Vladimir S. Gerdjikov

Institute of Nuclear Research

and Nuclear Energy

Bulgarian Academy of Sciences

Sofia 1784, BULGARIA

E-mail address: gerjikov@inrne.bas.bg

Nikolay A. Kostov

Institute of Nuclear Research

and Nuclear Energy

Bulgarian Academy of Sciences

Sofia 1784, BULGARIA

E-mail address: nakostov@inrne.bas.bg 